\title{
Implicit Vector Integral Equations Associated with Discontinuous Operators
}

\author{
Paolo Cubiotti ${ }^{1}$ and Jen-Chih Yao ${ }^{2,3}$ \\ ${ }^{1}$ Department of Mathematics and Computer Science, University of Messina, Viale F. Stagno d'Alcontres 31, 98166 Messina, Italy \\ ${ }^{2}$ Center for Fundamental Science, Kaohsiung Medical University, Kaohsiung 807, Taiwan \\ ${ }^{3}$ Department of Mathematics, King Abdulaziz University, P.O. Box 80203, Jeddah 21589, Saudi Arabia
}

Correspondence should be addressed to Jen-Chih Yao; yaojc@cc.kmu.edu.tw

Received 19 February 2014; Accepted 25 March 2014; Published 14 April 2014

Academic Editor: Chong Li

Copyright (C) 2014 P. Cubiotti and J.-C. Yao. This is an open access article distributed under the Creative Commons Attribution License, which permits unrestricted use, distribution, and reproduction in any medium, provided the original work is properly cited.

Let $I:=[0,1]$. We consider the vector integral equation $h(u(t))=f\left(t, \int g(t, z), u(z), d z\right)$ for a.e. $t \in I$, where $f: I \times J \rightarrow \mathbf{R}, g:$ $I \times I \rightarrow\left[0,+\infty\left[\right.\right.$, and $h: X \rightarrow \mathbf{R}$ are given functions and $X, J$ are suitable subsets of $\mathbf{R}^{n}$. We prove an existence result for solutions $u \in L^{s}\left(I, \mathbf{R}^{n}\right)$, where the continuity of $f$ with respect to the second variable is not assumed. More precisely, $f$ is assumed to be a.e. equal (with respect to second variable) to a function $f^{*}: I \times J \rightarrow \mathbf{R}$ which is almost everywhere continuous, where the involved null-measure sets should have a suitable geometry. It is easily seen that such a function $f$ can be discontinuous at each point $x \in J$. Our result, based on a very recent selection theorem, extends a previous result, valid for scalar case $n=1$.

\section{Introduction}

Let $I:=[0,1]$. Recently, in the paper [1], the following integral equation was studied: given $\lambda>0, f: I \times[0, \lambda] \rightarrow \mathbf{R}$, $g: I \times I \rightarrow[0,+\infty[, h:] 0,+\infty[\rightarrow \mathbf{R}$, and $s>1$; find $u \in L^{s}(I)$ such that

$$
h(u(t))=f\left(t, \int_{I} g(t, z) u(z) d z\right) \text { for a.e. } t \in I \text {. }
$$

In [1], the following existence result was proved, where, unlike other results in the field (see, for instance, the papers [2-5] and references therein, to which we also refer for motivations for studying (1)), the continuity of $f$ with respect to the second variable was not assumed.

Theorem 1 (Theorem 1 of [1]). Let $\lambda>0, A \subseteq] 0,+\infty[$ a closed interval, $h: A \rightarrow \mathbf{R}$ a continuous function, $f:$ $I \times[0, \lambda] \rightarrow \mathbf{R}$, and $g: I \times I \rightarrow[0,+\infty[$ two given functions. Let $s \in] 1,+\infty], \phi_{0} \in L^{j}(I)$, with $j>1$ and $j \geq s^{\prime}$ (the conjugate exponent of $s), \phi_{1} \in L^{s^{\prime}}(I)$, and $\beta \in L^{s}(I)$. Assume that (i) there exists a function $f^{*}: I \times[0, \lambda] \rightarrow \mathbf{R}$, two negligible sets $E_{1}, E_{2} \subseteq[0, \lambda]$, with $E_{2}$ closed, and a countable dense subset $D$ of $[0, \lambda]$, such that for all $x \in D$ the function $f^{*}(\cdot, x)$ is measurable, and for a.e. $t \in I$ one has

$$
\left\{x \in[0, \lambda]: f(t, x) \neq f^{*}(t, x)\right\} \subseteq E_{1},
$$

$\left\{x \in[0, \lambda]: f^{*}(t, \cdot)\right.$ is discontinuous at $\left.x\right\} \subseteq E_{2}$;

(ii) for all $z \in \operatorname{int}(h(A))$ (the interior of $h(A)$ ), one has int $h^{-1}(z)=\emptyset$;

(iii) if one puts, for all $t \in I$,

$$
v(t):=\underset{x \in[0, \lambda]}{\operatorname{essinf}} f(t, x), \quad z(t):=\underset{x \in[0, \lambda]}{\operatorname{ess} \sup } f(t, x),
$$

then for a.e. $t \in I$ one has

$$
[v(t), z(t)] \subseteq h(A), \quad \sup h^{-1}([v(t), z(t)]) \leq \beta(t) ;
$$


(iv) one has

$$
0<\left\|\phi_{0}\right\|_{L^{s^{\prime}(I)}} \leq \frac{\lambda}{\|\beta\|_{L^{s}(I)}} ;
$$

(v) for all $t \in I$, the function $g(t, \cdot)$ is measurable;

(vi) for a.e. $z \in I$, the function $g(\cdot, z)$ is continuous in $I$, differentiable in ]0,1[ and

$\left.g(t, z) \leq \phi_{0}(z), \quad 0<\frac{\partial g}{\partial t}(t, z) \leq \phi_{1}(z) \quad \forall t \in\right] 0,1[$

Then, there exists a solution $u \in L^{s}(I)$ to (1).

Of course, the main peculiarity of Theorem 1 resides in the kind of discontinuity that is allowed for $f$. Indeed, it is easy to construct examples of functions $f, g$, and $h$ satisfying the assumptions of Theorem 1 and such that for all $t \in I$ the function $f(t, \cdot)$ is discontinuous at all points $x \in[0, \lambda]$.

Theorem 1 extends a previous result (Theorem 1 of [6]), valid for the case where $f$ does not depend on $t$ explicitly. At this point, it is natural to ask if Theorem 1 above can be extended to the more general case where the function $u$ takes its values in the space $\mathbf{R}^{n}$. In this direction, we note that some results exist for the vector explicit equation

$$
u(t)=f\left(t, \int_{I} g(t, z) u(z) d z\right)
$$

(see $[7,8]$ ), while for the implicit equation (1) the problem is still unsolved.

The aim of this note is exactly to provide such an extension. In the following, if $n \in \mathbf{N}$ and $i \in\{1, \ldots, n\}$, we will denote by $P_{i}: \mathbf{R}^{n} \rightarrow \mathbf{R}$ the projection over the $i$ th axis. Moreover, we will denote by $m_{n}$ the $n$-dimensional Lebesgue measure over $\mathbf{R}^{n}$. If $\lambda:=\left(\lambda_{1}, \ldots, \lambda_{n}\right) \in \mathbf{R}^{n}$, with $\lambda_{i}>0$ for all $i=1, \ldots, n$, we will put $J_{\lambda}:=\prod_{i=1}^{n}\left[0, \lambda_{i}\right]$. Finally, if $n$ and $\lambda$ are as above, we will denote by $\mathscr{F}_{n, \lambda}$ the family of all subsets $F \subseteq J_{\lambda}$ such that there exist sets $F_{1}, F_{2}, \ldots, F_{n} \subseteq \mathbf{R}^{n}$, with $m_{1}\left(P_{i}\left(F_{i}\right)\right)=0$ for all $i=1 \ldots, n$, such that $F:=\bigcup_{i=1}^{n} F_{i}$. The following is our main result (where $\mathbf{R}_{n}^{+}$denotes the positive open orthant of $\mathbf{R}^{n}$, and $\operatorname{int}_{A}(B)$ is the interior of $B$ in $A$ ).

Theorem 2. Let $\lambda=\left(\lambda_{1}, \ldots, \lambda_{n}\right) \in \mathbf{R}_{+}^{n}$, and let $X \subseteq \mathbf{R}_{+}^{n}$ be a nonempty, closed, connected, and locally connected subset of $\mathbf{R}^{n}$, with inf $P_{i}(X)>0$ for all $i=1, \ldots, n$. Let $h: X \rightarrow \mathbf{R}$ be a continuous function and $f: I \times J_{\lambda} \rightarrow \mathbf{R}$ and $g: I \times I \rightarrow$ $[0,+\infty[$ two given functions. Let $s \in] 1,+\infty], \phi_{0} \in L^{j}(I)$, with $j>1$ and $j \geq s^{\prime}, \phi_{1} \in L^{s^{\prime}}(I)$, and $\beta \in L^{s}\left(I, \mathbf{R}^{n}\right)$. Finally, let $D$ be a countable dense subset of $J_{\lambda}$.

Assume that there exists a function $f^{*}: I \times J_{\lambda} \rightarrow \mathbf{R}$ and two sets $E, F \in \mathscr{F}_{n, \lambda}$, with $F$ closed, such that

(i) for all $x \in D$ the function $f^{*}(\cdot, x)$ is measurable;

(ii) for a.e. $t \in I$ one has

$$
\left\{x \in J_{\lambda}: f(t, x) \neq f^{*}(t, x)\right\} \subseteq E,
$$

$\left\{x \in J_{\lambda}: f^{*}(t, \cdot)\right.$ is discontinuous at $\left.x\right\} \subseteq F$.
Moreover, assume that

(iii) $\operatorname{int}_{X}\left(h^{-1}(t)\right)=\emptyset$, for all $t \in \operatorname{int}_{\mathbf{R}}(h(X))$;

(iv) if one puts, for all $t \in I$;

$$
v(t):=\underset{x \in J_{\lambda}}{\operatorname{essinf}} f(t, x), \quad z(t):=\underset{x \in \mathrm{J}_{\lambda}}{\operatorname{ess} \sup } f(t, x),
$$

then for a.e. $t \in I$ and all $i=1, \ldots, n$ one has

$$
\begin{aligned}
& {[v(t), z(t)] \subseteq h(X),} \\
& \quad \sup P_{i}\left(h^{-1}([v(t), z(t)])\right) \leq \beta_{i}(t),
\end{aligned}
$$

(where $\beta_{i}: I \rightarrow \mathbf{R}$ denotes the ith component of the function $\beta$ );

(v) one has

$$
0<\left\|\phi_{0}\right\|_{L^{s^{\prime}(I)}} \leq \min _{1 \leq i \leq n} \frac{\lambda_{i}}{\left\|\beta_{i}\right\|_{L^{s}(I)}} ;
$$

(vi) for all $t \in I$, the function $g(t, \cdot)$ is measurable;

(vii) for a.e. $z \in I$, the function $g(\cdot, z)$ is continuous in $I$, differentiable in ]0, 1 [ and

$$
\left.g(t, z) \leq \phi_{0}(z), \quad 0<\frac{\partial g}{\partial t}(t, z) \leq \phi_{1}(z) \quad \forall t \in\right] 0,1[.
$$

Then, there exists $u \in L^{s}\left(I, \mathbf{R}^{n}\right)$ such that

$$
h(u(t))=f\left(t, \int_{I} g(t, z) u(z) d z\right) \quad \text { for a.e. } t \in I \text {. }
$$

Theorem 2 will be proved as an application of the following selection theorem, recently proved in [9], which we now state for the reader's convenience (in the following, if $S$ is a topological space, we will denote by $\mathscr{B}(S)$ the Borel family of S).

Theorem 3 (Theorem 2.2 of [9]). Let $T$ and $X_{1}, X_{2}, \ldots X_{k}$ be complete separable metric spaces, with $k \in \mathbf{N}$, and let $X:=$ $\prod_{j=1}^{k} X_{j}$ (endowed with the product topology). Let $\mu, \psi_{1}, \ldots, \psi_{k}$ be positive regular Borel measures over $T, X_{1}, X_{2}, \ldots X_{k}$, respectively, with $\mu$ finite and $\psi_{1}, \ldots, \psi_{k} \sigma$-finite.

Let $S$ be a separable metric space, and let $F: T \times X \rightarrow 2^{S}$ be a multifunction with nonempty complete values. Finally, let $E \subseteq X$ be a given set, and, for each $i \in\{1, \ldots, k\}$, let $P_{*, i}: X \rightarrow$ $X_{i}$ be the projection over $X_{i}$. Assume that

(i) the multifunction $F$ is $\mathscr{T}_{\mu} \otimes \mathscr{B}\left(X_{1}\right) \otimes \cdots \otimes \mathscr{B}\left(X_{k}\right)$ measurable (where $\mathscr{T}_{\mu}$ denotes the completion of the Borel $\sigma$-algebra $\mathscr{B}(T)$ of $T$ with respect to the measure $\mu)$;

(ii) for a.e. $t \in T$, one has

$$
\left\{x:=\left(x_{1}, \ldots, x_{k}\right) \in X: F(t, \cdot)\right.
$$

is not lower semicontinuous at $x\} \subseteq E$. 
Then, there exist sets $Q_{1}, \ldots, Q_{k}$, with $Q_{i} \in \mathscr{B}\left(X_{i}\right)$ and $\psi_{i}\left(Q_{i}\right)=0$ for all $i=1, \ldots, k$, and a function $\phi: T \times X \rightarrow S$ such that

(a) $\phi(t, x) \in F(t, x)$ for all $(t, x) \in T \times X$;

(b) for all $x:=\left(x_{1}, x_{2}, \ldots, x_{k}\right) \in X \backslash\left[\left(\bigcup_{i=1}^{k} P_{*, i}^{-1}\left(Q_{i}\right)\right) \cup E\right]$, the function $\phi(\cdot, x)$ is $\mathscr{T}_{\mu}$-measurable over $T$;

(c) for a.e. $t \in T$, one has

$$
\begin{aligned}
& \left\{x:=\left(x_{1}, x_{2}, \ldots, x_{k}\right) \in X: \phi(t, \cdot)\right. \\
& \quad \text { is discontinuous at } x\} \subseteq E \cup\left[\bigcup_{i=1}^{k} P_{*, i}^{-1}\left(Q_{i}\right)\right] .
\end{aligned}
$$

The proof of Theorem 2 will be given in Section 2. Further, we will point out some counterexamples to possible improvements of Theorem 2.

\section{Proof of Theorem 2}

Before giving the proof of Theorem 2, we fix some notations. If $n \in \mathbf{N}$, the space $\mathbf{R}^{n}$ will be considered with its Euclidean norm $\|\cdot\|_{n}$. Moreover, if $x \in \mathbf{R}^{n}$ and $r>0$, we put

$$
\begin{aligned}
& B(x, r):=\left\{v \in \mathbf{R}^{n}:\|v-x\|_{n}<r\right\}, \\
& \bar{B}(x, r):=\left\{v \in \mathbf{R}^{n}:\|v-x\|_{n} \leq r\right\} .
\end{aligned}
$$

If $p \in[1,+\infty]$, the space $L^{p}\left(I, \mathbf{R}^{n}\right)$ will be considered with the usual norm

$$
\begin{aligned}
& \|u\|_{L^{p}\left(I, \mathbf{R}^{n}\right)}:=\left(\int_{I}\|u(t)\|_{n}^{p} d t\right)^{1 / p} \quad \text { if } p<+\infty \\
& \|u\|_{L^{\infty}\left(I, \mathbf{R}^{n}\right)}:=\text { ess } \sup \|u(t)\|_{n} \quad \text { if } p=+\infty
\end{aligned}
$$

As usual, we put $L^{p}(I):=L^{p}(I, \mathbf{R})$. For the basic definitions and facts about multifunctions, we refer to [10].

Proof of Theorem 2. Without loss of generality, we can assume that (8) and (10) hold for all $t \in I$. Moreover, we can suppose that $j<+\infty$. Firstly, we prove that the functions $v$ and $z$ are measurable. Observe that, by assumption (ii), for all $t \in I$, one has

$$
v(t)=\inf _{x \in J_{\lambda} \backslash F} f^{*}(t, x), \quad z(t)=\sup _{x \in J_{\lambda} \backslash F} f^{*}(t, x) .
$$

To see this, fix $t \in I$, and let $\psi(t):=\sup _{x \in J_{\lambda} \backslash F} f^{*}(t, x)$. Since $m_{n}(E \cup F)=0$ we get

$$
z(t) \leq \sup _{x \in J_{\lambda} \backslash(E \cup F)} f(t, x)=\sup _{x \in J_{\lambda} \backslash(E \cup F)} f^{*}(t, x) \leq \psi(t) .
$$

Now, assume that $z(t)<\psi(t)$. Hence, there is $x^{*} \in J_{\lambda} \backslash F$ such that $f^{*}\left(t, x^{*}\right)>z(t)$. Since the function $f^{*}(t, \cdot)$ is continuous at $x^{*}$, there exist $\delta, \varepsilon>0$ such that

$$
f^{*}(t, x)>z(t)+\varepsilon \quad \forall x \in J_{\lambda} \cap B\left(x^{*}, \delta\right) .
$$

Since $m_{n}\left(J_{\lambda} \cap B\left(x^{*}, \delta\right)\right)>0$, we get

$$
z(t):=\underset{x \in J_{\lambda}}{\operatorname{ess} \sup } f(t, x)=\underset{x \in J_{\lambda}}{\operatorname{ess} \sup } f^{*}(t, x) \geq z(t)+\varepsilon,
$$

which is absurd. Therefore, the second equality in (18) is proved. The first one can be checked in analogous way.

Since $F$ is closed, it can be easily checked that the set $D \cap\left(J_{\lambda} \backslash F\right)$ is nonempty, countable, and dense in $\left(J_{\lambda} \backslash F\right)$. Consequently, by Lemma at page 198 of [11], the function $\left.f^{*}\right|_{I \times\left(J_{\lambda} \backslash F\right)}$ is $\mathscr{L}(I) \otimes \mathscr{B}\left(J_{\lambda} \backslash F\right)$-measurable (where $\mathscr{L}(I)$ denotes the family of all Lebesgue-measurable subsets of $I$ ). By (18) and Lemma III.39 of [12], it follows that the functions $v$ and $z$ are measurable over $I$, as claimed.

By assumption (iii) and Theorem 2.4 di [13], there exists a set $Y \subseteq X$ such that $h(Y)=h(X)$ and the function $\left.h\right|_{Y}$ is open (it carries open subsets of $Y$ onto open subsets of $h(X)=h(Y))$. Consequently, the multifunction $T: h(X) \rightarrow$ $2^{Y}$ defined by putting, for each $s \in h(X)$,

$$
T(s):=h^{-1}(s) \cap Y,
$$

is lower semicontinuous in $h(X)$ with nonempty values. Let $f_{0}: I \times J_{\lambda} \rightarrow \mathbf{R}$ be defined by putting, for all $(t, x) \in I \times J_{\lambda}$,

$$
f_{0}(t, x)= \begin{cases}f^{*}(t, x) & \text { if } x \notin F \\ z(t) & \text { if } x \in F .\end{cases}
$$

Clearly, the function $f_{0}$ is $\mathscr{L}(I) \otimes \mathscr{B}\left(J_{\lambda}\right)$-measurable and, by (18), one has

$$
v(t) \leq f_{0}(t, x) \leq z(t) \quad \forall(t, x) \in I \times J_{\lambda} .
$$

Moreover, assumption (ii) and the closedness of $F$ imply that for all $t \in I$ one has

$$
\left\{x \in J_{\lambda}: f_{0}(t, \cdot) \text { is discontinuous at } x\right\} \subseteq F \text {. }
$$

Let $G: I \times J_{\lambda} \rightarrow 2^{Y}$ be the multifunction defined by setting, for each $(t, x) \in I \times J_{\lambda}$,

$$
G(t, x):=T\left(f_{0}(t, x)\right)=h^{-1}\left(f_{0}(t, x)\right) \cap Y .
$$

Observe that $G$ is well-defined since for all $(t, x) \in I \times J_{\lambda}$ one has

$$
f_{0}(t, x) \in[v(t), z(t)] \subseteq h(X) .
$$

Moreover, by the lower semicontinuity of $T$ and by (25), for all $t \in I$, we get

$\left\{x \in J_{\lambda}: G(t, \cdot)\right.$ is not lower semicontinuous at $\left.x\right\} \subseteq F$.

Let $\Psi: I \times J_{\lambda} \rightarrow 2^{\mathbf{R}^{n}}$ (more precisely, $\Psi: I \times J_{\lambda} \rightarrow 2^{\bar{Y}}$ ) be the multifunction defined by putting, for each $(t, x) \in I \times J_{\lambda}$, $\Psi(t, x):=\overline{G(t, x)}$. By $(28)$, for all $t \in[0,1]$, we get

$\left\{x \in J_{\lambda}: \Psi(t, \cdot)\right.$ is not lower semicontinuous at $\left.x\right\} \subseteq F$.

Moreover, the values of $\Psi$ are closed (in $\mathbf{R}^{n}$ ) subsets of $X$. 
Since $f_{0}$ is $\mathscr{L}(I) \otimes \mathscr{B}\left(J_{\lambda}\right)$-measurable and $T$ is lower semicontinuous, by Proposition 13.2.1 of [10] the multifunction $G$ is $\mathscr{L}(I) \otimes \mathscr{B}\left(J_{\lambda}\right)$-weakly measurable. That is, for each set $\Omega \subseteq Y$, with $\Omega$ open in the relative topology of $Y$, the set

$$
G^{-}(\Omega):=\left\{(t, x) \in I \times J_{\lambda}: G(t, x) \cap \Omega \neq \emptyset\right\}
$$

belongs to $\mathscr{L}(I) \otimes \mathscr{B}\left(J_{\lambda}\right)$. By Proposition 2.6 and Theorem 3.5 of [14], the multifunction $\Psi$ is $\mathscr{L}(I) \otimes \mathscr{B}\left(J_{\lambda}\right)$-measurable.

Since $F \in \mathscr{F}_{n, \lambda}$, there exist sets $F_{1}, F_{2}, \ldots, F_{n} \subseteq \mathbf{R}^{n}$ such that $F=\bigcup_{i=1}^{n} F_{i}$ and $m_{1}\left(P_{i}\left(F_{i}\right)\right)=0$ for all $i=1 \ldots, n$. By Theorem 3, there exist sets $Q_{1}, Q_{2}, \ldots, Q_{n} \subseteq \mathbf{R}$, with $Q_{i} \in$ $\mathscr{B}\left(\left[0, \lambda_{i}\right]\right)$ and $m_{1}\left(Q_{i}\right)=0$ for all $i=1, \ldots, n$, and a function $\psi:[0,1] \times J_{\lambda} \rightarrow \mathbf{R}^{n}$ such that

(a) for all $(t, x) \in I \times J_{\lambda}$ one has $\psi(t, x) \in \Psi(t, x)$;

(b) for all $x \in J_{\lambda} \backslash\left[\bigcup_{i=1}^{n}\left(P_{i}^{-1}\left(Q_{i}\right) \cup F_{i}\right)\right]$, the function $\psi(\cdot, x)$ is $\mathscr{L}(I)$-measurable;

(c) for a.e. $t \in I$, one has

$$
\begin{gathered}
\left\{x \in J_{\lambda}: \psi(t, \cdot) \text { is discontinuous at } x\right\} \\
\subseteq\left[\bigcup_{i=1}^{n}\left(P_{i}^{-1}\left(Q_{i}\right) \cup F_{i}\right)\right] \cap J_{\lambda} .
\end{gathered}
$$

Since $X$ is closed and $h$ is continuous, for all $(t, x) \in I \times$ $J_{\lambda}$, the set $h^{-1}\left(f_{0}(t, x)\right)$ is closed in $\mathbf{R}^{n}$. Consequently, for all $(t, x) \in I \times J_{\lambda}$, we get

$$
\begin{aligned}
\psi(t, x) \in \Psi(t, x) & =\overline{h^{-1}\left(f_{0}(t, x)\right) \cap Y} \subseteq \overline{h^{-1}\left(f_{0}(t, x)\right)} \\
& =h^{-1}\left(f_{0}(t, x)\right) .
\end{aligned}
$$

Now, let

$$
\alpha:=\min _{1 \leq i \leq n} \inf P_{i}(X)>0 .
$$

By (27), (32), and assumption (iv) we get

$$
\psi(t, x) \in \prod_{i=1}^{n}\left[\alpha, \beta_{i}(t)\right] \quad \forall(t, x) \in I \times J_{\lambda} .
$$

Let $\psi_{1}: I \times \mathbf{R}^{n} \rightarrow \mathbf{R}^{n}$ be defined by putting

$$
\psi_{1}(t, x)= \begin{cases}\psi(t, x) & \text { if } x \in J_{\lambda} \\ \beta(t) & \text { if } x \in \mathbf{R}^{n} \backslash J_{\lambda} .\end{cases}
$$

By (34) and (35) we easily get

$$
\psi_{1}(t, x) \in \prod_{i=1}^{n}\left[\alpha, \beta_{i}(t)\right] \quad \forall(t, x) \in I \times \mathbf{R}^{n} .
$$

Let

$$
\Lambda:=\left[\bigcup_{i=1}^{n}\left(P_{i}^{-1}\left(Q_{i}\right) \cup F_{i}\right)\right] \cap J_{\lambda},
$$

and let $D_{0}$ be any countable dense subset of $J_{\lambda} \backslash \Lambda$. Since $m_{n}(\Lambda)=0$, it is easily seen that $D_{0}$ is dense in $J_{\lambda}$. Let $D_{1}$ be any countable dense subset of $\mathbf{R}^{n} \backslash J_{\lambda}$. Then, the set $D_{2}:=D_{0} \cup D_{1}$ is countable and dense in $\mathbf{R}^{n}$, and for all $x \in D_{2}$ the function $\psi_{1}(\cdot, x)$ is measurable by the above construction. Moreover, taking into account (31), for all $t \in I$, one has

$$
\begin{aligned}
\{x & \left.\in \mathbf{R}^{n}: \psi_{1}(t, \cdot) \text { is discontinuous at } x\right\} \\
& \subseteq\left[\bigcup_{i=1}^{n}\left(P_{i}^{-1}\left(Q_{i}\right) \cup P_{i}^{-1}\left(\left\{0, \lambda_{i}\right\}\right) \cup F_{i}\right)\right] \cap J_{\lambda} .
\end{aligned}
$$

Let $H:[0,1] \times \mathbf{R}^{n} \rightarrow 2^{\mathbf{R}^{n}}$ be defined by putting, for all $(t, x) \epsilon$ $[0,1] \times \mathbf{R}^{n}$,

$$
H(t, x)=\bigcap_{m \in \mathbf{N}} \overline{\operatorname{conv}} \overline{\left(\bigcup_{\substack{y \in D_{2} \\\|x-y\|_{n} \leq 1 / m}}\left\{\psi_{1}(t, y)\right\}\right)},
$$

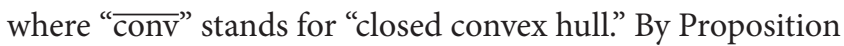
2 of [8], taking into account (36) and (38), we have that

(a) $H$ has nonempty closed convex values;

(b) for all $x \in \mathbf{R}^{n}$, the multifunction $H(\cdot, x)$ is measurable;

(c) for all $t \in I$, the multifunction $H(t, \cdot)$ has closed graph;

(d) for all $t \in I$, one has

$H(t, x)=\left\{\psi_{1}(t, x)\right\}$

$$
\begin{gathered}
\forall x \in \mathbf{R}^{n} \backslash\left(\left[\bigcup _ { i = 1 } ^ { n } \left(P_{i}^{-1}\left(Q_{i}\right) \cup P_{i}^{-1}\left(\left\{0, \lambda_{i}\right\}\right)\right.\right.\right. \\
\left.\left.\left.\cup F_{i}\right)\right] \cap J_{\lambda}\right) .
\end{gathered}
$$

Moreover, by (36), we have

$$
H(t, x) \subseteq \prod_{i=1}^{n}\left[\alpha, \beta_{i}(t)\right] \quad \forall(t, x) \in I \times \mathbf{R}^{n} .
$$

Now we want to apply Theorem 1 of [15], choosing $T=[0,1]$, $X=Y=\mathbf{R}^{n}, p=s, q=j^{\prime}, V=L^{s}\left(I, \mathbf{R}^{n}\right), \Psi(u)=u, r=$ $\|\beta\|_{L^{s}\left(I, \mathbf{R}^{n}\right)}, \varphi \equiv+\infty, F=H$, and

$$
\Phi(u)(t)=\int_{I} g(t, z) u(z) d z .
$$

To this aim, we can argue as in [8]. In particular, observe the following.

(a) $\Phi\left(L^{s}\left(I, \mathbf{R}^{n}\right)\right) \subseteq C^{0}\left(I, \mathbf{R}^{n}\right)$. This follows easily from our assumptions (vi) and (vii) and the classical Lebesgue's dominated convergence theorem.

(b) If $v \in L^{s}\left(I, \mathbf{R}^{n}\right)$ and $\left\{v^{k}\right\}$ is a sequence in $L^{s}\left(I, \mathbf{R}^{n}\right)$, weakly convergent to $v$ in $L^{j^{\prime}}\left(I, \mathbf{R}^{n}\right)$, then the sequence $\left\{\Phi\left(v^{k}\right)\right\}$ converges to $\Phi(v)$ strongly in $L^{1}\left(I, \mathbf{R}^{n}\right)$. This follows by Theorem 2 at page 359 of [16], since $g$ is $j$ th power summable in $I \times I$ (note that $g$ is measurable on $I \times I$ by the classical Scorza-Dragoni theorem; see [17] or also [11]). 
(c) By (41), the function

$$
h_{0}: t \in I \longrightarrow \sup _{x \in \mathbf{R}^{n}} \inf _{y \in H(t, x)}\|y\|_{n}
$$

belongs to $L^{s}(I)$ and $\left\|h_{0}\right\|_{L^{s}(I)} \leq\|\beta\|_{L^{s}\left(I, \mathbf{R}^{n}\right)}$.

Therefore, taking into account the above construction, all the assumptions of Theorem 1 of [15] are satisfied. Consequently, there exists a function $u^{*} \in L^{s}\left(I, \mathbf{R}^{n}\right)$ and a set $K_{1} \subseteq I$, with $m_{1}(K)=0$, such that

$$
u^{*}(t) \in H\left(t, \Phi\left(u^{*}\right)(t)\right) \subseteq \prod_{i=1}^{n}\left[\alpha, \beta_{i}(t)\right] \quad \forall t \in I \backslash K_{1} .
$$

That is,

$$
\begin{array}{r}
u^{*}(t) \in H\left(t, \int_{I} g(t, z) u^{*}(z) d z\right) \subseteq \prod_{i=1}^{n}\left[\alpha, \beta_{i}(t)\right] \\
\forall t \in I \backslash K_{1} .
\end{array}
$$

We now prove that the function $u^{*}$ satisfies our conclusion. To this aim, observe that, since $E \in \mathscr{F}_{n, \lambda}$, there exist sets $E_{1}, E_{2}, \ldots, E_{n} \subseteq \mathbf{R}^{n}$, with $m_{1}\left(P_{i}\left(E_{i}\right)\right)=0$ for all $i=1, \ldots, n$, such that $E:=\bigcup_{i=1}^{n} E_{i}$.

Fix $i \in\{1, \ldots, n\}$. Let $\gamma_{i}: I \rightarrow \mathbf{R}$ be the function

$$
\gamma_{i}(t):=P_{i}\left(\Phi\left(u^{*}\right)(t)\right)=\int_{I} g(t, z) u_{i}^{*}(z) d z .
$$

By (45) we get

$$
u_{i}^{*}(t) \in\left[\alpha, \beta_{i}(t)\right] \quad \forall t \in I \backslash K_{1} ;
$$

hence for all $t \in I$ we get the inequality

$$
\begin{aligned}
0 & \leq \gamma_{i}(t) \leq\left\|\phi_{0}\right\|_{L^{s^{\prime}(I)}}\left\|u^{*}\right\|_{L^{s}(I)} \\
& \leq \frac{\lambda_{i}}{\left\|\beta_{i}\right\|_{L^{s}(I)}} \cdot\left\|\beta_{i}\right\|_{s}=\lambda_{i},
\end{aligned}
$$

hence $\gamma_{i}(I) \subseteq\left[0, \lambda_{i}\right]$. By (vi), (vii), and (45) we have that $\gamma_{i}$ is strictly increasing. Moreover, by Lemma 2.2. at page 226 of [18] we get

$$
\left.\gamma_{i}^{\prime}(t)=\int_{I} \frac{\partial g}{\partial t}(t, z) u_{i}^{*}(z) d z>0 \quad \forall t \in\right] 0,1[.
$$

Consequently, by Theorem 2 of [19], the function $\gamma_{i}^{-1}$ is absolutely continuous. By Theorem 18.25 of [20], the set

$$
W_{i}:=\gamma_{i}^{-1}\left[\left(P_{i}\left(E_{i} \cup F_{i}\right) \cup Q_{i} \cup\left\{0, \lambda_{i}\right\}\right) \cap \gamma_{i}(I)\right]
$$

has null Lebesgue measure. Now, put

$$
\Omega:=\left(\bigcup_{i=1}^{n} W_{i}\right) \cup K_{1} .
$$

Of course, $m_{1}(\Omega)=0$. Choose any point $t^{*} \in I \backslash \Omega$. Since $t^{*} \notin K_{1}$, by (45) we get

$$
u^{*}\left(t^{*}\right) \in H\left(t^{*}, \Phi\left(u^{*}\right)\left(t^{*}\right)\right) .
$$

For each $i \in\{1, \ldots, n\}$, since $t^{*} \notin W_{i}$, taking into account (48), we have

$$
\gamma_{i}\left(t^{*}\right) \in\left[0, \lambda_{i}\right] \backslash\left[P_{i}\left(E_{i} \cup F_{i}\right) \cup\left\{0, \lambda_{i}\right\} \cup Q_{i}\right] .
$$

Therefore, $\Phi\left(u^{*}\right)\left(t^{*}\right) \in J_{\lambda}$ and for all $i \in\{1, \ldots, n\}$ we have

$$
\Phi\left(u^{*}\right)\left(t^{*}\right) \notin\left[\left(E_{i} \cup F_{i}\right) \cup P_{i}^{-1}\left(\left\{0, \lambda_{i}\right\}\right) \cup P_{i}^{-1}\left(Q_{i}\right)\right] .
$$

Consequently, we get

$$
\begin{aligned}
& \Phi\left(u^{*}\right)\left(t^{*}\right) \\
& \quad \in J_{\lambda} \backslash \bigcup_{i=1}^{n}\left[\left(E_{i} \cup F_{i}\right) \cup P_{i}^{-1}\left(\left\{0, \lambda_{i}\right\}\right) \cup P_{i}^{-1}\left(Q_{i}\right)\right] .
\end{aligned}
$$

By (40) we get

$$
\begin{aligned}
u^{*}\left(t^{*}\right) \in H\left(t^{*}, \Phi\left(u^{*}\right)\left(t^{*}\right)\right) & =\left\{\psi_{1}\left(t^{*}, \Phi\left(u^{*}\right)\left(t^{*}\right)\right)\right\} \\
& =\left\{\psi\left(t^{*}, \Phi\left(u^{*}\right)\left(t^{*}\right)\right)\right\} .
\end{aligned}
$$

By (32) and (56) we get

$$
u^{*}\left(t^{*}\right) \in h^{-1}\left(f_{0}\left(t^{*}, \Phi\left(u^{*}\right)\left(t^{*}\right)\right),\right.
$$

hence

$$
h\left(u^{*}\left(t^{*}\right)\right)=f_{0}\left(t^{*}, \Phi\left(u^{*}\right)\left(t^{*}\right)\right) .
$$

Since $\Phi\left(u^{*}\right)\left(t^{*}\right) \notin E \cup F$, we get

$$
\begin{aligned}
h\left(u^{*}\left(t^{*}\right)\right) & =f^{*}\left(t^{*}, \Phi\left(u^{*}\right)\left(t^{*}\right)\right) \\
& =f\left(t^{*}, \Phi\left(u^{*}\right)\left(t^{*}\right)\right) \\
& =f\left(t^{*}, \int_{i} g\left(t^{*}, z\right) u^{*}(z) d z\right) .
\end{aligned}
$$

This completes the proof.

Remark 4. Of course, a function $f$ satisfying the assumptions of Theorem 2 can be discontinuous at each point $x \in J_{\lambda}$. The Example at the end of [8] shows that, in the statement of Theorem 2, none of the sets $E$ and $F$ can be assumed to depend on $t$. Moreover, the Example at the end of [6] shows that the second inequality in assumption (vii) cannot be weakened by assuming that

$$
0 \leq \frac{\partial g}{\partial t}(t, z) \leq \phi_{1}(z) .
$$

\section{Conflict of Interests}

The authors declare that there is no conflict of interests regarding the publication of this paper.

\section{Acknowledgment}

This research was partially supported by Grant NSC 1032923-E-037-001-MY3. 


\section{References}

[1] G. Anello and P. Cubiotti, "Non-autonomous implicit integral equations with discontinuous right-hand side," Commentationes Mathematicae Universitatis Carolinae, vol. 45, no. 3, pp. 417-429, 2004.

[2] J. Banaś and Z. Knap, "Integrable solutions of a functionalintegral equation," Revista Matemática de la Universidad Complutense de Madrid, vol. 2, no. 1, pp. 31-38, 1989.

[3] G. Emmanuele, "About the existence of integrable solutions of a functional-integral equation," Revista Matemática de la Universidad Complutense de Madrid, vol. 4, no. 1, pp. 65-69, 1991.

[4] G. Emmanuele, "Integrable solutions of a functional-integral equation," Journal of Integral Equations and Applications, vol. 4, no. 1, pp. 89-94, 1992.

[5] M. Fečkan, "Nonnegative solutions of nonlinear integral equations," Commentationes Mathematicae Universitatis Carolinae, vol. 36, no. 4, pp. 615-627, 1995.

[6] F. Cammaroto and P. Cubiotti, "Implicit integral equations with discontinuous right-hand side," Commentationes Mathematicae Universitatis Carolinae, vol. 38, no. 2, pp. 241-246, 1997.

[7] F. Cammaroto and P. Cubiotti, "Vector integral equations with discontinuous right-hand side," Commentationes Mathematicae Universitatis Carolinae, vol. 40, no. 3, pp. 483-490, 1999.

[8] P. Cubiotti, "Non-autonomous vector integral equations with discontinuous right-hand side," Commentationes Mathematicae Universitatis Carolinae, vol. 42, no. 2, pp. 319-329, 2001.

[9] P. Cubiotti and J. C. Yao, "Two-point problem for vector differential inclusions with discontinuous right-hand side," Applicable Analysis, 2013.

[10] E. Klein and A. C. Thompson, Theory of Correspondences, John Wiley \& Sons, New York, NY, USA, 1984.

[11] A. Kucia, "Scorza Dragoni type theorems," Polska Akademia Nauk. Fundamenta Mathematicae, vol. 138, no. 3, pp. 197-203, 1991.

[12] C. Castaing and M. Valadier, Convex Analysis and Measurable Multifunctions, Springer, Berlin, Germany, 1977.

[13] B. Ricceri, "Sur la semi-continuité inférieure de certaines multifonctions," Comptes Rendus des Séances de l'Académie des Sciences. Série I. Mathématique, vol. 294, no. 7, pp. 265-267, 1982.

[14] C. J. Himmelberg, "Measurable relations," Polska Akademia Nauk. Fundamenta Mathematicae, vol. 87, pp. 53-72, 1975.

[15] O. N. Ricceri and B. Ricceri, "An existence theorem for inclusions of the type $\Psi(u)(t) \in F(t, \Phi(u)(t))$ and application to a multivalued boundary value problem," Applicable Analysis, vol. 38, no. 4, pp. 259-270, 1990.

[16] L. V. Kantorovich and G. P. Akilov, Functional Analysis in Normed Spaces, Pergamon Press, Oxford, UK, 1964.

[17] G. Scorza Dragoni, "Un teorema sulle funzioni continue rispetto ad una e misurabili rispetto ad unaltra variabile," Rendiconti del Seminario Matematico della Università di Padova, vol. 17, pp. 102-106, 1948.

[18] S. Lang, Real and Functional Analysis, Springer, New York, NY, USA, 1993.

[19] A. Villani, "On Lusin's condition for the inverse function," Rendiconti del Circolo Matematico di Palermo II, vol. 33, no. 3, pp. 331-335, 1984.

[20] E. Hewitt and K. Stromberg, Real and Abstract Analysis, Springer, Berlin, Germany, 1975. 


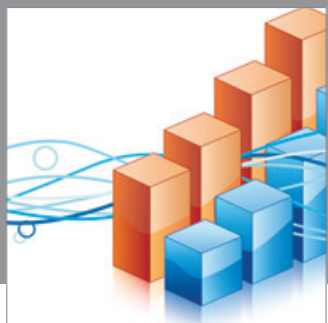

Advances in

Operations Research

mansans

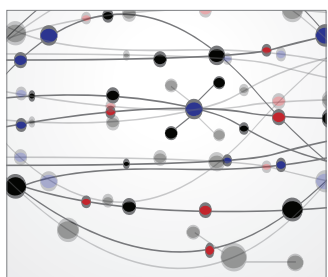

The Scientific World Journal
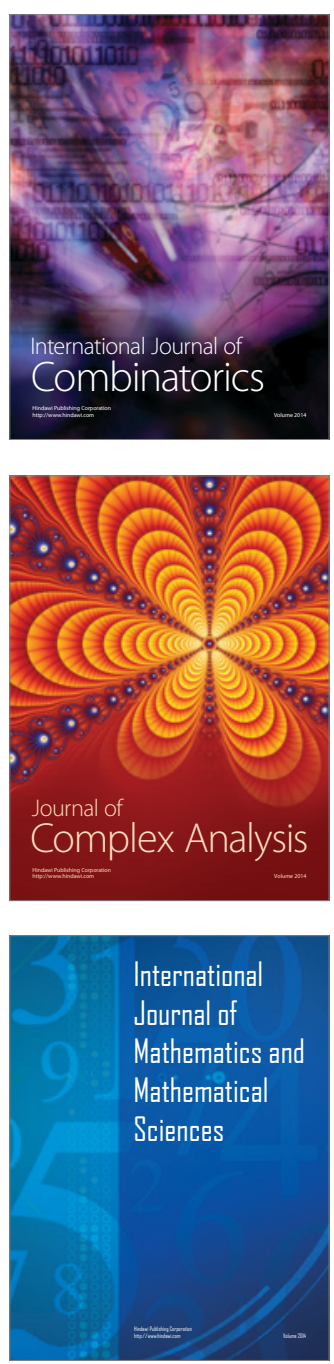
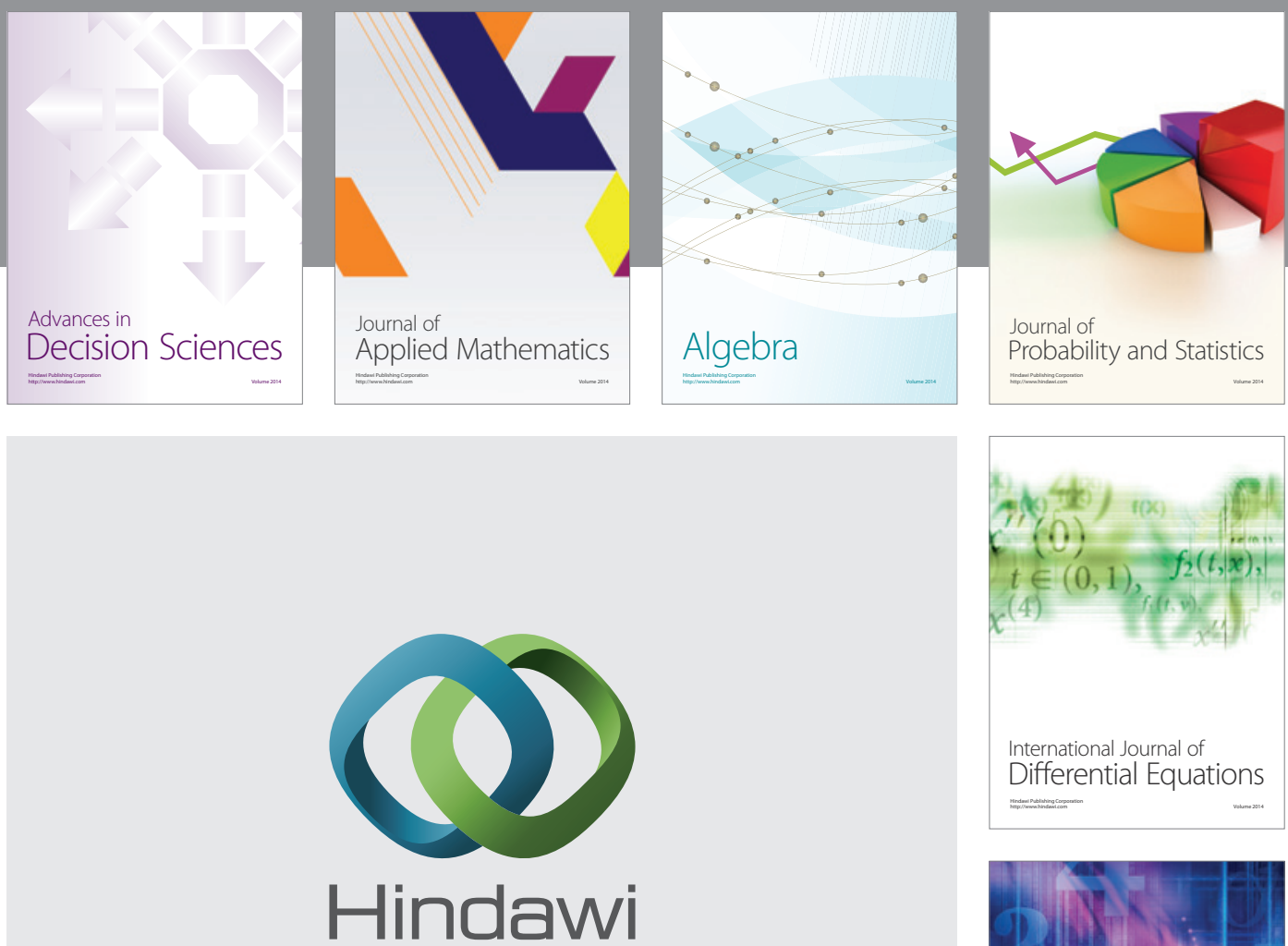

Submit your manuscripts at http://www.hindawi.com
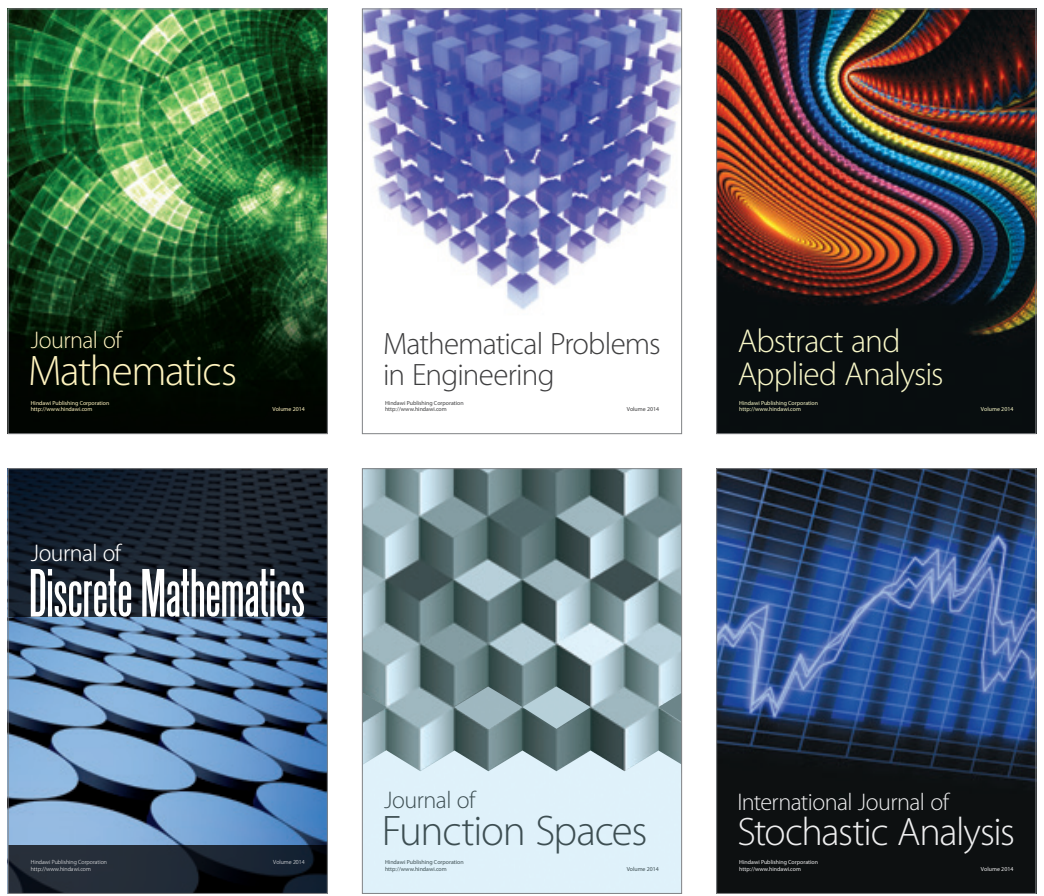

Journal of

Function Spaces

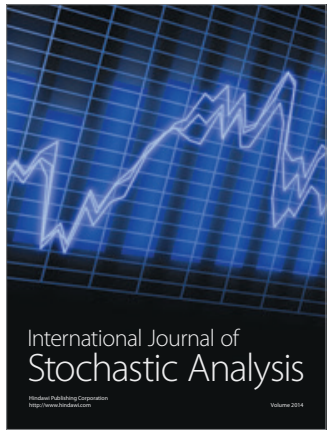

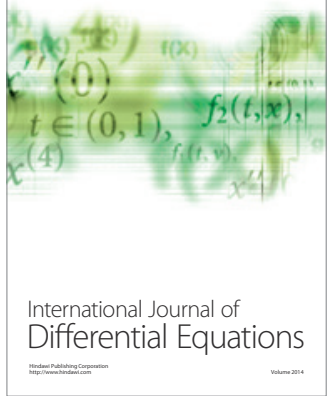
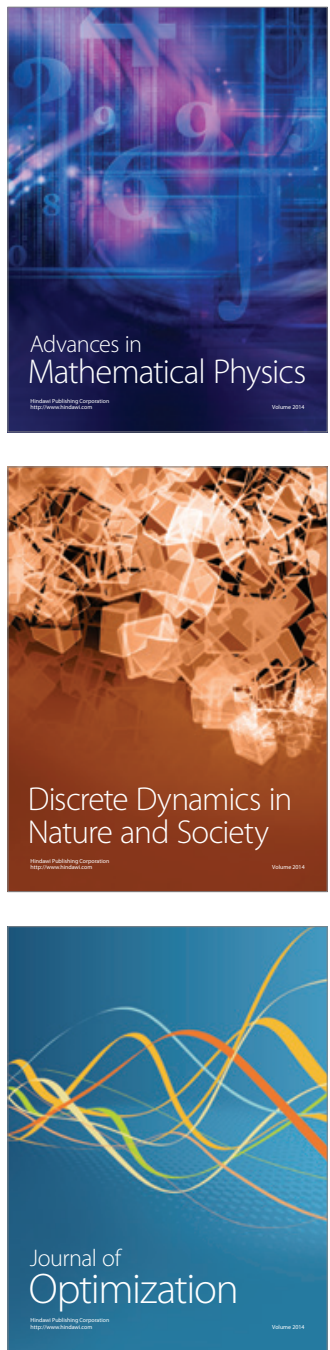